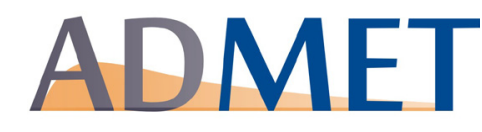

Open Access : ISSN: 1848-7718

http://www.pub.iapchem.org/ojs/index.php/admet/index

Book Review

\title{
Finding a needle in a haystack
}

\section{Zoran Mandić}

Faculty of Chemical Engineering and Technology, University of Zagreb, Marulićev trg 19, HR-10000 Zagreb, Croatia E-mail: zmandic@fkit.hr; Tel.: +385-1-4597-164; Fax: +385-1-3733-640

Received: December 20, 2013; Revised: January 10, 2013; Published: January 14, 2013

Absorption and Drug Development. Solubility, Permeability and Charge State. $2^{\text {nd }}$ Edition. Alex Avdeef, John Wiley \& Sons Inc. 2012.

In my early days in pharmaceutical research I was summoned into the boss's office where a meeting was taking place. I came right into a discussion about the determination of the partition coefficients of two substances of potential significance to the company. "This is a notorious time wasting", I muttered to myself while trying to avoid being drawn into the discussion. What would have been more important was the synthesis of new chemical entities, which certainly had very good potential to become new blockbusters on the drugs market, especially those originating from my flask.

Little did I know at that time that the issue of accurate prediction of drug absorption in the early stages of drug discovery would become so important. It was only some time later, when a colleague threw a paper in front of me claiming excitedly that it was something we could and should do, that I gave the subject a second thought. It was one of a series of Alex Avdeef's papers about physico-chemical profiling of drug substances including the potentiometric determination of octanol-water partition coefficients. It took no time for the colleague of mine to assemble a potentiometric titration device from scraps and pieces of old, discarded equipment, and off we went...

Now, almost two decades later, while flicking through the pages of the second edition of Alex's book Absorption and Drug Development, I find myself astonished by how much the concept of drug absorption and its importance has progressed into a scientific discipline without which no contemporary pharmaceutical research could be considered. The author describes in a very simple way four key physicochemical processes, i.e. ionization, solubility, lipophilicity and permeability, which are of relevance to the absorption of most orally administered drugs, and explains various relationships among them. By the application of simple scientific concepts of physico-chemical profiling and continuously placing them in the drug development setting, readers can easily gain deep understanding of their significance. Physicochemical parameters such as $\mathrm{pKa}, \log P$ or $\log S$ are no longer perceived by medicinal chemists or formulation and pre-formulation engineers as "good" or "bad" numbers, but provide valuable information revealing and predicting the whole pathway and the fate of the drug from its administration to entering the bloodstream. 
This book is based on the author's many years of experience and on the experience of many other scientists who have tackled the subject of drug absorption and given it the significance it deserves. If the author's goal was to "examine the components of multimechanistic processes related to charge state.... with the aim of advancing improved strategies for in vitro assays related to drug absorption", then there is no shadow of doubt that he achieved it. However, the core strength of Absorption and Drug Development is that it provides solid ground on which different worlds, embodied in the labs of medicinal chemists, pharmaceutical scientists, analytical chemists and even pharma managers, can understand each other and together rationalize the results obtained and their consequences.

The book is conceptually organized into ten chapters. Chapter 1 is an introductory chapter that places the topic appropriately in the pharmaceutical research context. Chapter 2 gives a physico-chemical model of drug permeation based on the $\mathrm{pH}$-partition hypothesis and Fick's laws of diffusion. This is followed by a description of biological environments relevant to drug absorption.

Molecular ionization and the acid-base properties of drugs are dealt with in Chapter 3. This Chapter is the most comprehensive coverage of the theory and practical aspects of $p K a$ determination by potentiometric techniques I have ever encountered in textbooks of physical chemistry. From the beginning of the Chapter readers learn the importance of $\mathrm{pKa}$ as a molecular property defining the charge state of drug in various parts of the body. The thermodynamics of ionization reactions are explained followed by a description of the practical aspects of $\mathrm{pKa}$ measurements that should be taken into account while performing $\mathrm{pKa}$ determinations with a glass electrode. Data handling and data presentation are introduced with the emphasis on avoiding errors and false results. Particular attention is paid to coping with challenges and obstacles that might be encountered in pKa determination of low solubility substances of pharmaceutical interest. All topics are accompanied with many practical examples and tutorials.

Chapters 4 and 5 describe lipophilicity as a molecular property of considerable importance in drug absorption. Octanol-water partitioning (Chapter 4) and "biomimetic" liposome-water partitioning (Chapter 5) and their determinations are presented. Special emphasis is placed on ion-pair partitioning and its significance for drug absorption. An attempt is made to define new concepts and explain some of the anomalies in lipophilicity results.

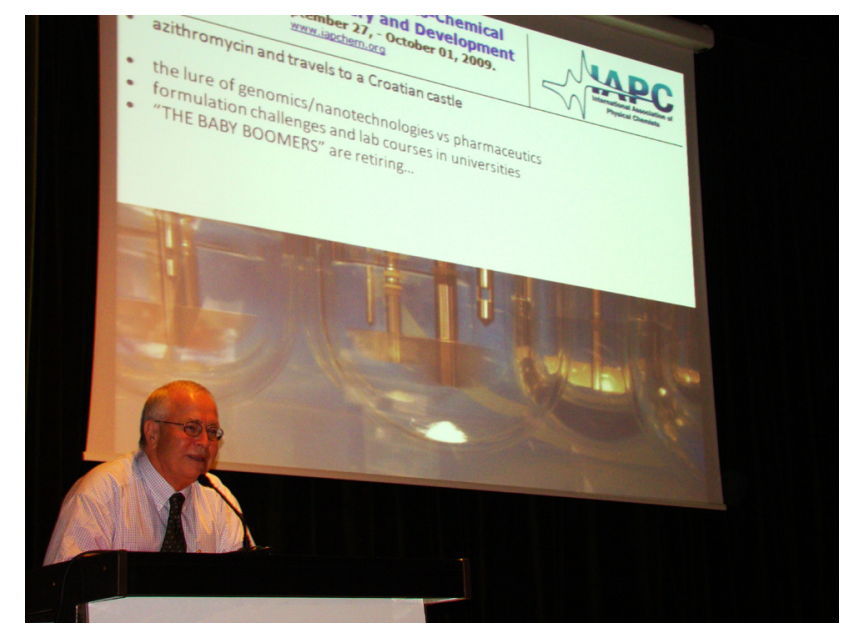

Alex Avdeef giving a lecture at the $1^{\text {st }}$ World Conference on Physico-Chemical Methods in Drug Discovery and Development, organized by IAPC in Rovinj, Croatia, 2009. 
An in-depth discussion of solubility and its measurements is presented in Chapter 6. Important concepts are illustrated with several case studies. $\mathrm{pH}$-dependent solubility profiles are evaluated and highthroughput solubility measurements are tackled.

Chapters 7-9 deal with the concepts of permeability. The practical and theoretical background of prediction of human jejunal permeability and human intestinal absorption by in-vitro assays, most notably by PAMPA, is illustrated in a broad sense (Chapter 7). Cell-based assays, Caco-2 and MDCK are explored in Chapter 8. The idea is to develop protocols and advanced data analysis for inter-laboratory cell-based transport studies. Finally, fundamental concepts of permeability measurements using endothelial cell models of the blood-brain barrier are covered in Chapter 9.

Throughout the book the rules and laws of physical chemistry are followed. They are explained in a simple way but with sufficient detail for readers to gain a complete insight into the topics. Furthermore, this book gathers together a huge collection of relevant physico-chemical parameters determined by the author and his co-workers or found in other literature. More than 900 pKa values, 350 octanol/water partition coefficients, 100 liposome/water partition coefficients, 60 solubility constants, 300 permeability coefficients, and almost 700 Caco-2/MDCK permeability coefficients as well as 200 permeability values related to the blood-brain barrier can be found in addition to many other parameters of pharmaceutical interest.

This book is essential literature on the desk of the pharmaceutical scientist and is highly recommended to students and others interested in the topic of drug absorption.

${ }^{\circ} 2013$ by the authors; licensee IAPC, Zagreb, Croatia. This article is an open-access article distributed under the terms and conditions of the Creative Commons Attribution license (http://creativecommons.org/licenses/by/3.0/) (cc)) EY 